\title{
Neutrino Mass, Mixing and Oscillations
}

\author{
Hiroshi Nunokawa \\ Instituto de Física Gleb Wataghin, Universidade Estadual de Campinas - UNICAMP, \\ 13083-970 Campinas, Brazil \\ E-mail: nunokawa@ifi.unicamp.br
}

Received 7 January, 2000

\begin{abstract}
Present data from various neutrino experiments are strongly suggesting the existence of neutrino mass and flavor mixing. We review the present status of neutrino oscillation search and various neutrino anomalies and their possible interpretations in terms of oscillation induced by neutrino mass and flavor mixing.
\end{abstract}

\section{Introduction}

Neutrinos have been playing an important role in the evolution of our understanding of elementary particles and could have some significant impact on the evolution of the universe. We have strong motivations to search for non-zero neutrino mass both from theoretical and experimental aspects. Various observational data which are indicating the existence of neutrino mass and flavor mixing are piling up. In fact neutrino physics and astrophysics have become very active field now. In the last decade, many new neutrino experiments have been performed and various important results were obtained.

Neutrinos are regarded to have spin $1 / 2$, charge zero, helicity -1 and known to exist in three flavors, i.e., electron, muon and tau although the direct detection of tau neutrinos is still in progress [1]. Fourth neutrino, if exist, must be sterile (or electroweak singlet) in order to be consistent with the observed $Z$ decay width at LEP experiment [2]. The combined LEP results give the number of light (not heavier than the half of $Z$ boson mass) neutrino as,

$$
N_{\nu}=2.993 \pm 0.011
$$

Various experimental data coming from atmospheric neutrino observations [3-9], solar neutrinos [1014], and LSND experiment [15] are strongly suggesting the existence of neutrino mass and flavor mixing. There is also some cosmological indication of neutrino masses. It has been discussed [16] that in order to explain the large scale structures of our universe, certain amount of hot dark matter with mass in the few $\mathrm{eV}$ range is needed. Massive neutrinos are the most natural candidate for such particles.

The organizations of this review is as follows. In Sec. II we review the formulation of neutrino oscillation, which is the most powerful tool to search for very tiny neutrino masses. In Sec. III and IV we review at- mospheric and solar neutrino observations, respectively. In Sec. $\mathrm{V}$ we review the present status of laboratory search for neutrino mass and oscillation. In the above sections III-V, we describe the possible interpretations as well as constraints from the various observations in terms of neutrino oscillations in the simplest two flavor scheme. In Sec. VI we discuss how the picture we discussed in Sections III-V could be incorporated into three or four flavor scheme. Sec. VII is devoted for conclusions and outlook.

\section{Neutrino Oscillation Formal- ism}

\section{II.1 Neutrino Oscillation in Vacuum}

The idea of neutrino oscillation was first introduced by Pontecorvo [17] for neutrino and anti-neutrino system, similar to $K^{0}-\bar{K}^{0}$ oscillation. Oscillation between different flavor was first considered by Maki, Nakagawa and Sakata [18]. The basic idea is as follows. If neutrinos are massive, in general the mass eigenstates and flavor (or weak interaction) eigenstates do not coincide but they are related by a unitary transformation similar to the Cabibbo-Kobayashi-Maskawa (CKM) [19] mixing matrix in the quark sector. In the neutrino sector such mixing matrix is called Maki-Nakagawa-Sakata (MNS) matrix [18].

For two generations, we can describe this explicitly as follows,

$$
\left[\begin{array}{l}
\nu_{e} \\
\nu_{\mu}
\end{array}\right]=\left[\begin{array}{cc}
\cos \theta & \sin \theta \\
-\sin \theta & \cos \theta
\end{array}\right]\left[\begin{array}{l}
\nu_{1} \\
\nu_{2}
\end{array}\right]
$$

where $\nu_{e}, \nu_{\mu}$ and $\nu_{1}, \nu_{2}$ are the weak and mass eigenstates, respectively. If mass of neutrinos are different $\left(m_{1} \neq m_{2}\right)$ a non-trivial phase between the two states 
can develop and this can lead to neutrino oscillation between different flavors [18].

The oscillation probability can be described as

$$
\begin{gathered}
P\left(\nu_{e} \rightarrow \nu_{\mu}\right)=\sin ^{2} 2 \theta \sin ^{2}\left[\frac{\Delta m^{2}}{4 E} L\right] \\
=\sin ^{2} 2 \theta \sin ^{2}\left[1.27\left(\frac{\Delta m^{2}}{\mathrm{eV}^{2}}\right)\left(\frac{\mathrm{MeV}}{E}\right)\left(\frac{L}{m}\right)\right],
\end{gathered}
$$

where $\Delta m^{2} \equiv m_{2}^{2}-m_{1}^{2}$ is the mass squared difference of the two mass eigenvalues, $E$ is the neutrino energy and $L$ is the distance neutrino travels. From above eq. we see that the oscillation length in vacuum is given by,

$$
L_{o s c}=\frac{\pi}{1.27}\left[\frac{E_{\nu}}{\mathrm{MeV}}\right]\left[\frac{\mathrm{eV}^{2}}{\Delta m^{2}}\right] \mathrm{m} .
$$

For three generation, we can describe the neutrino mixing as,

$$
\left[\begin{array}{l}
\nu_{e} \\
\nu_{\mu} \\
\nu_{\tau}
\end{array}\right]=U\left[\begin{array}{l}
\nu_{1} \\
\nu_{2} \\
\nu_{3}
\end{array}\right]
$$

where $\nu_{\alpha}(\alpha=e, \mu, \tau)$ and $\nu_{i}(i=1,2,3)$ are the weak and mass eigenstates, respectively and the MNS matrix $U$ can be parametrized in a same way as the CKM matrix in the quark sector [2],

$$
\begin{gathered}
U= \\
c_{12} c_{13} \\
{\left[\begin{array}{ccc}
s_{12} c_{13} & s_{13} e^{-i \delta} \\
-s_{12} c_{23}-c_{12} s_{23} s_{13} e^{i \delta} & c_{12} c_{23}-s_{12} s_{23} s_{13} e^{i \delta} & s_{23} c_{13} \\
s_{12} s_{23}-c_{12} c_{23} s_{13} e^{i \delta} & -c_{12} s_{23}-s_{12} c_{23} s_{13} e^{i \delta} & c_{23} c_{13}
\end{array}\right],}
\end{gathered}
$$

where $c_{i j}=\cos \theta_{i j}, s_{i j}=\sin \theta_{i j}$ and $\delta$ is the CP violating phase. We note that if neutrinos are of Majorana type the MNS matrix have more CP phases which can not be rotated away by the redefinitions of the neutrino fields. See, e.g., Ref. [20] about the CP phases for Majorana neutrinos.

In vacuum, the oscillation probability can be written as,

$$
\begin{aligned}
& P\left(\nu_{\alpha} \rightarrow \nu_{\beta}\right)=\delta_{\alpha \beta} \\
& -4 \sum_{i<j} \operatorname{Re}\left[U_{\alpha i} U_{\alpha j}^{*} U_{\beta i}^{*} U_{\beta j}\right] \sin ^{2}\left[\frac{\Delta m_{i j}^{2}}{4 E} L\right] \\
& +2 \sum_{i<j} \operatorname{Im}\left[U_{\alpha i} U_{\alpha j}^{*} U_{\beta i} U_{\beta j}^{*}\right] \sin \left[\frac{\Delta m_{i j}^{2}}{2 E} L\right] .
\end{aligned}
$$

The last term gives rise to possible CP and/or T violation in the neutrino oscillation [21].

\section{II.2 Neutrino Oscillation in Matter}

Here, for simplicity, we consider neutrino oscillation in matter mainly for the case with two flavor.

Evolution equation of neutrino system of $\nu_{e}$ and $\nu_{x}$ $(x=\mu$ or $\tau)$ in matter can be written as,

$$
i \frac{d}{d t}\left[\begin{array}{l}
\nu_{e} \\
\nu_{x}
\end{array}\right]=H\left[\begin{array}{l}
\nu_{e} \\
\nu_{x}
\end{array}\right]
$$

where

$$
H=\left[\begin{array}{cc}
-\frac{\Delta m^{2}}{4 E} \cos 2 \theta+\sqrt{2} G_{F} N_{e} & \frac{\Delta m^{2}}{4 E} \sin 2 \theta \\
\frac{\Delta m^{2}}{4 E} \sin 2 \theta & -\frac{\Delta m^{2}}{4 E} \cos 2 \theta
\end{array}\right]
$$

Here, $N_{e}$ is the electron number density in matter and $G_{F}$ is the Fermi constant.

Mixing angle in matter, which diagonalize the Hamiltonian in eq. (10) is given by,

$$
\sin ^{2} 2 \theta_{m}=\frac{\sin ^{2} 2 \theta}{\left(\cos 2 \theta-\frac{\sqrt{2} G_{F} N_{e} E}{\Delta m^{2}}\right)^{2}+\sin ^{2} 2 \theta}
$$

From above equation we see that even if the vacuum mixing angle is very small, the mixing angle in matter can be maximal if the following condition (resonance condition),

$$
\frac{\Delta m^{2}}{2 E} \cos 2 \theta=\sqrt{2} G_{F} N_{e}
$$

is satisfied. If the density is varying along the neutrino trajectory and if, at the position where the above condition is met, the following condition (adiabaticity condition),

$$
\left.\frac{1}{N_{e}} \frac{d N_{e}}{d r}\right|_{r e s} \ll \frac{\Delta m^{2} \sin ^{2} 2 \theta}{E \cos 2 \theta}
$$

is also satisfied, electron neutrino can convert completely into another type (mu or tau) of neutrino. This phenomena is called the matter enhanced resonant conversion or the MSW effect [22] and can provide solutions to the solar neutrino problem [23] (see Sec. IV).

For three flavor, as in the case of vacuum (see eq. (8)) in general, oscillation probability depends on six parameters, i.e., two independent $\Delta m_{i j}^{2}$, three mixing angles $\theta_{i j}$ and one CP phase $\delta$. However, if there is a hierarchy between the mass, and if only one $\Delta m \equiv$ $\Delta m_{12}^{2}\left(\ll \Delta m_{13}^{2} \simeq \Delta m_{23}^{2}\right)$ is relevant for the resonant conversion in the sun, the probability of finding $\nu_{e}$ after the resonance $P_{e e}^{3 \nu}$ can be given by [24],

$$
\begin{gathered}
P_{e e}^{3 \nu}\left(\Delta m^{2}, \theta_{12}, \theta_{13}\right)=\sin ^{4} \theta_{13} \\
+\left.\cos ^{4} \theta_{13} P_{e e}^{2 \nu}\left(\Delta m^{2}, \theta_{12}\right)\right|_{N_{e} \rightarrow N_{e} \cos ^{2} \theta_{13}},
\end{gathered}
$$

where $P_{e e}^{2 \nu}$ is the corresponding probability for the case of two flavor. 


\section{Atmospheric anomaly}

At present the most strong indication of neutrino oscillation is coming from the very impressive results obtained by the Super-Kamiokande (SK) experiment on atmospheric neutrino observation $[7,8,9]$.

So called atmospheric neutrinos are mainly produced by the following decay chain of charged pions which are produced by primary cosmic ray,

$$
\begin{array}{cc}
\pi^{+} \rightarrow \mu^{+}+\nu_{\mu}, & \mu^{+} \rightarrow \bar{\nu}_{\mu}+e^{+}+\nu_{e} \\
\pi^{-} \rightarrow \mu^{-}+\bar{\nu}_{\mu}, & \mu^{-} \rightarrow \nu_{\mu}+e^{-}+\bar{\nu}_{e} .
\end{array}
$$

The flavor ratio of the flux, $R(\mu / e) \equiv\left(\nu_{\mu}+\overline{\overline{\nu_{\mu}}}\right) /\left(\nu_{e}+\right.$ $\left.\overline{\nu_{e}}\right)$, which is approximately 2 for lower energy neutrinos $(\sim \mathrm{GeV})$ and larger for higher energy neutrinos, can be calculable with an uncertainty about $5 \%$.

In the Super-Kamiokande detector, atmospheric neutrino induced events are classified into several types. If neutrino interactions occur inside the detector, the events are classified, by energy, into sub-GeV (when visible energy $E_{\text {vis }}<1.33 \mathrm{GeV}$ ) or multi-GeV events (when $E_{v i s}>1.33 \mathrm{GeV}$ ). They have both $e$-like and $\mu^{-}$ like events, which are induced by $\nu_{e}\left(\right.$ or $\bar{\nu}_{e}$ ) and $\nu_{\mu}$ (or $\bar{\nu}_{\mu}$ ), respectively. In sub-GeV and multi-GeV samples, the observed events are further classified into various types, single ring events, two ring events (include $\pi^{0}$ events), multi-ring events, fully contained (FC) events, partially contained $(\mathrm{PC})$ events, etc.

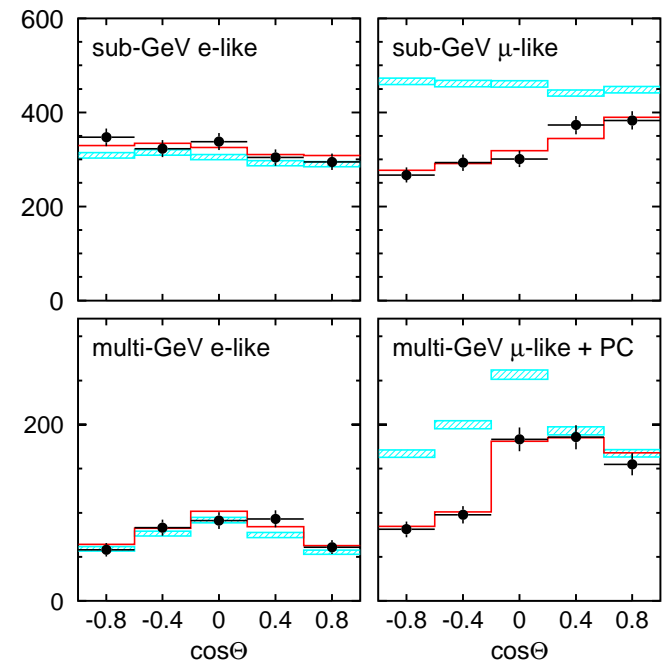

Figure 1. Angular distributions for $e$-like (left) and $\mu$-like (right) events, for sub-GeV (top) and multi-GeV (bottom) samples. The bars show the MC no-oscillation prediction with statistical errors, and the line shows the oscillation prediction for the best-fit parameters, $\sin ^{2} 2 \theta=1.0$ and $\Delta m^{2}=3.5 \times 10^{-3} \mathrm{eV}^{2}$. Taken from Ref. [8].

If neutrino interactions occur not inside the detector but in the rock below the detector, they are classified as upward going muons. This can apply only for the events induced by muon neutrinos since electrons produced by electron neutrinos would be immediately absorbed by the matter and can not reach the detector.

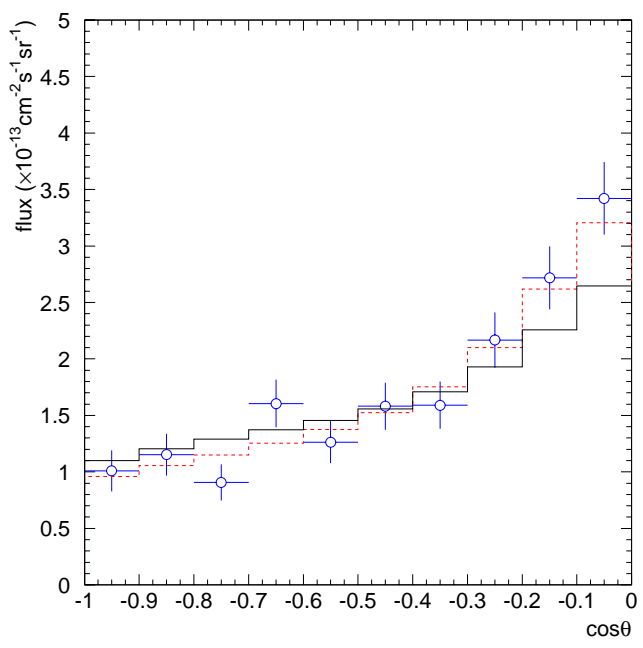

Figure 2. Angular distribution of through-going upward muons, as a function of $\cos \theta$. The circles represent the data, the solid line represents the normalized no-oscillation flux prediction, and the dashed line represents the best fit prediction for oscillations. Taken from Ref. [8].

For the SK sub-GeV sample, with 848 days exposure,

$$
\frac{R(\mu / e)_{\text {data }}}{R(\mu / e)_{\text {no-osc }}}=0.68 \pm 0.02 \text { (stat) } \pm 0.05 \text { (syst.) }
$$

For the SK multi-GeV sample,

$$
\frac{R(\mu / e)_{\text {data }}}{R(\mu / e)_{\text {no-osc }}}=0.68 \pm 0.04 \text { (stat) } \pm 0.08 \text { (syst.) }
$$

The above results are consistent with other atmospheric neutrino experiments IMB [3], Kamiokande [4] and Soudan2 [5], Macro [6] (except for Frejus [25] and NUSEX [26] which had the largest errors).

In addition to the significant overall deficit in the flavor ratio, Super-Kamiokande experiment is observing, clear zenith angle dependent deficit of muon neutrinos which is strongly implying that muon neutrinos are oscillating into some other flavor of neutrinos. 
The most plausible mechanism of such conversion is provided by neutrino oscillation between $\nu_{\mu}$ and $\nu_{\tau}$ induced by mass and mixing with $\Delta m^{2} \sim 10^{-3}-10^{-2}$ $\mathrm{eV}^{2}$ and $\sin ^{2} 2 \theta \gtrsim 0.8$.

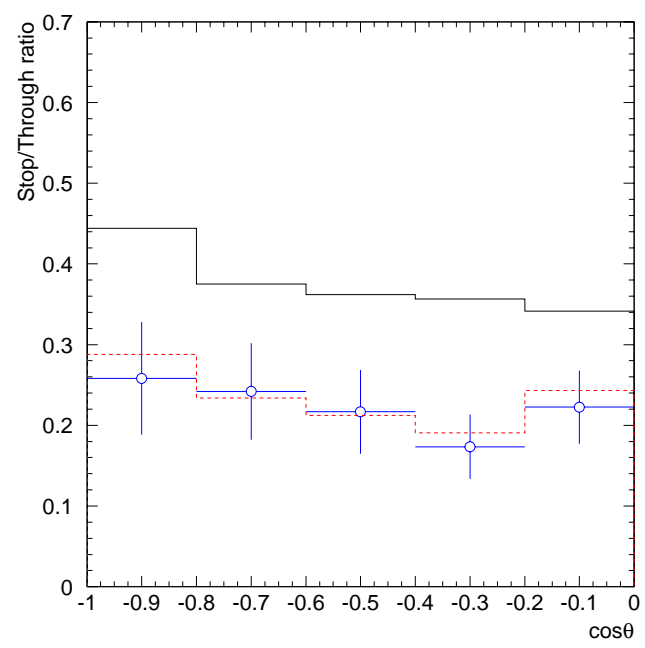

Figure 3. Same as in Fig. 2 but for stopping muons. Taken from Ref. [8].

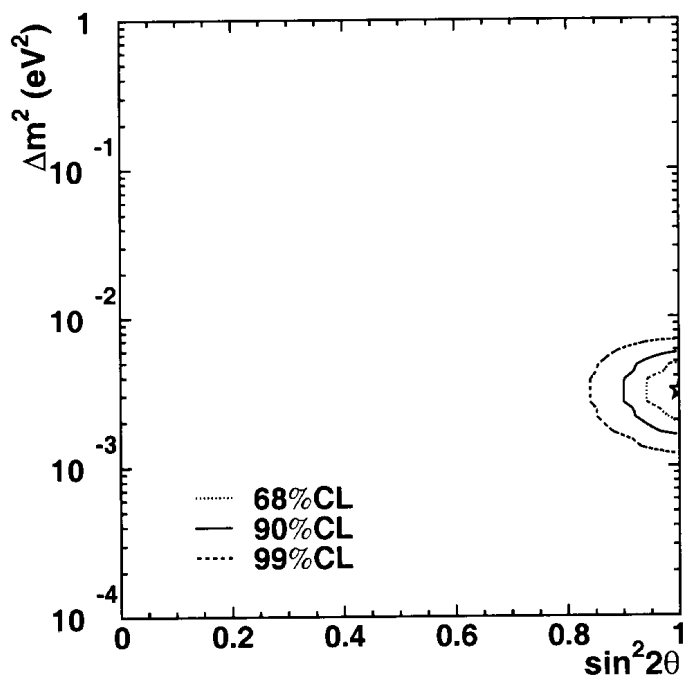

Figure 4. Allowed region for $\sin ^{2} 2 \theta$ and $\Delta m^{2}$ using the combined information from FC, PC and upward-going muons. Taken from Ref. [8].

In Fig. 1 we show the observed zenith angle dependence taken from Ref. [8]. We see that the $\mu$-like events of sub-GeV as well as multi-GeV have strong zenith angle dependence where the MC predictions are rather symmetric around $\cos \theta=0$. In the same graph, the best fitted theoretical predictions with neutrino oscillation hypothesis $\nu_{\mu} \leftrightarrow \nu_{\tau}$ are also plotted. We see that these curves explain the observed data very well. On the other hand, $e$-like events do not show any significant zenith angle distortion with respect to the prediction which implies that there is no significant oscillation between $\nu_{e}$ and other neutrino flavors.

In Fig. 4 we show the region of parameters which can explain the observed atmospheric data obtained by the Super-Kamiokande experiment[7].

We note that from the preliminary data of the angular dependence of the events with higher energy (multi$\mathrm{GeV}$ and upward going muons data) and the observed $\pi^{0}$ events the Super-Kamiokande collaboration is excluding $\nu_{\mu}-\nu_{s}$ oscillation hypothesis at $99 \%$ C.L. [9].

\section{Solar neutrino problem}

The chain of nuclear fusion reactions in the sun results in net production of one ${ }^{4} \mathrm{He}$ nucleus and two neutrinos out of four protons as,

$$
4 p \rightarrow \alpha+2 e^{+}+2 \nu_{e}
$$

The real situation in the sun is, however, a bit more complicated; it organizes itself as several branches of nuclear reaction network as described in Table 3.1 of Ref. [23]. In Fig. 5 we show the solar neutrino spectrum expected in the standard solar model [23].

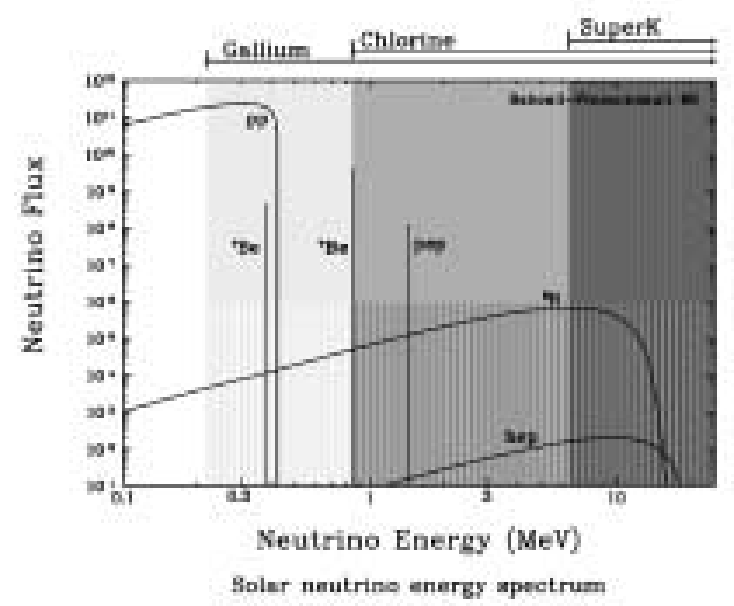

Figure 5. Solar neutrino spectrum. Taken from Ref. [23]. 
All the solar neutrino experiments, i.e., Homestake [10], SAGE [11], GALLEX [12], Kamiokande [13] and Super-Kamiokande [14] have been observing roughly only half or less neutrinos compared to the prediction by the standard solar model (SSM) [23, 27]. In Table 1 , we show the observed solar neutrino event rates and in Table 2, we show the corresponding predictions from the SSM by Bahcall and Pinsonneault [27].
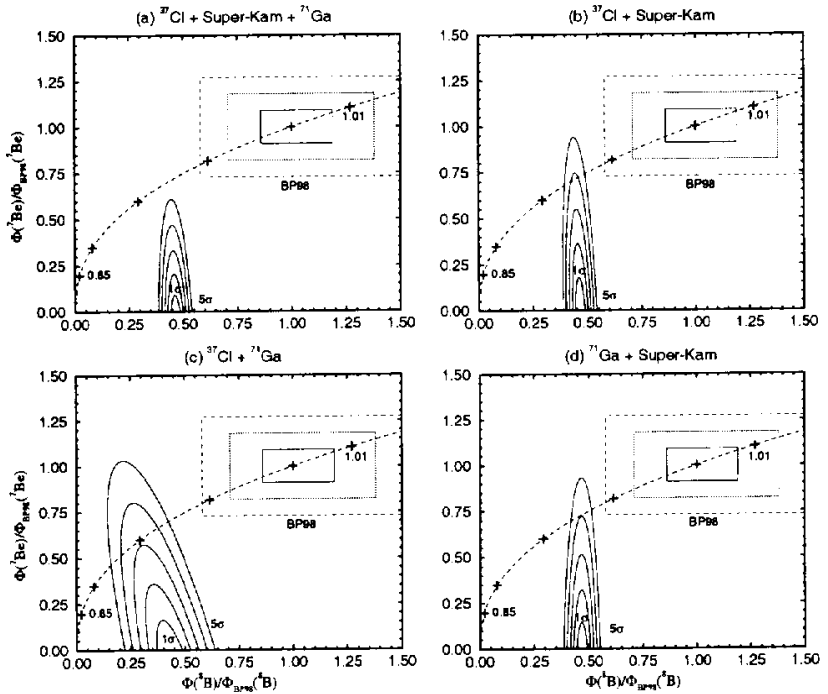

Figure 6. Contour plot of the $\chi^{2}$ values in the $\Phi_{8_{B}}-\Phi_{7_{B}}$ plane for different combinations of the solar neutrino experiments. The solid curves correspond to $1 \sigma$ to $5 \sigma$, with step size 1 , from inside to outside. We also indicate the 1,2 and $3 \sigma$ theoretical range predicted by BP98, by the solid, dotted and dashed lines, respectively. Along the dashed curve, $\phi^{7} B e=\left(\phi^{8} B\right)^{10 / 24}$, the crosses indicate, from left to right, the point where the central temperatures are $0.85,0.9,0.95$, $0.98,1$ and 1.01 with respect to the prediction by the SSM. Taken from Ref. [29].

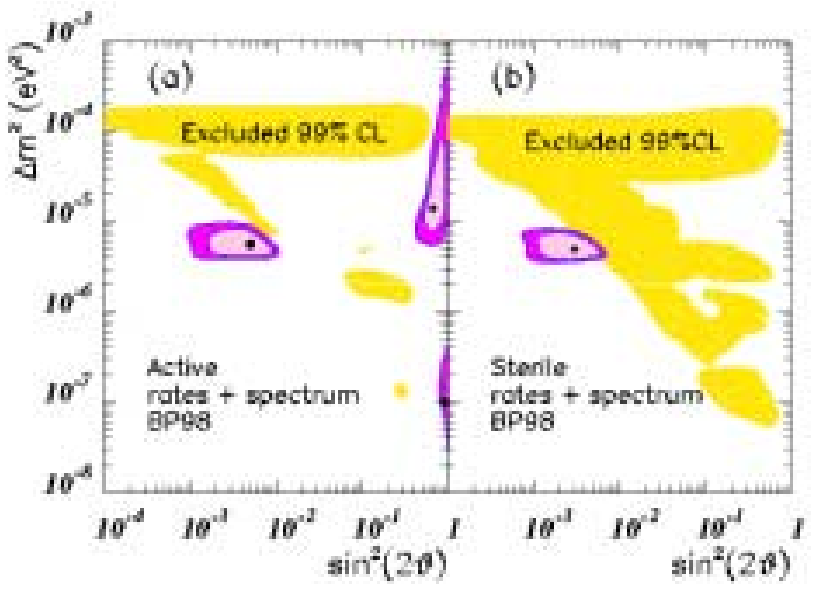

Figure 7. Allowed region for $\sin ^{2} 2 \theta$ and $\Delta m^{2}$ which can explain the observed solar neutrino data. Taken from Ref. [31].
Table 1. Observed solar neutrino event rates. The quoted errors are at $1 \sigma$.

\begin{tabular}{ccr}
\hline Experiment & Data \pm (stat. $) \pm$ (syst.) & Ref. \\
\hline Homestake & $2.56 \pm 0.16 \pm 0.15 \mathrm{SNU}$ & {$[10]$} \\
SAGE & $69.9_{-7.7-4.1}^{+8.0+3} \mathrm{SNU}$ & {$[11]$} \\
GALLEX & $76.4 \pm 6.3_{-4.9}^{+4.5} \mathrm{SNU}$ & {$[12]$} \\
Kamiokande & $(2.80 \pm 0.19 \pm 0.33) \times 10^{6} \mathrm{~cm}^{-2} \mathrm{~s}^{-1}[13]$ \\
SuperK & $\left(2.44 \pm 0.05_{-0.06}^{+0.09}\right) \times 10^{6} \mathrm{~cm}^{-2} \mathrm{~s}^{-1}[14]$ \\
\hline
\end{tabular}

Table 2. Predictions from the reference standard solar model [27]. The quoted errors are at $1 \sigma$.

\begin{tabular}{ccc}
\hline $\mathrm{Cl}$ & $7.7_{-1.0}^{+1.2}$ & $\mathrm{SNU}$ \\
$\mathrm{Ga}$ & $129_{-6}^{+8}$ & $\mathrm{SNU}$ \\
${ }^{8} \mathrm{~B}$ & $\left(5.15_{-0.72}^{+0.98}\right) \times 10^{6} \mathrm{~cm}^{-2} \mathrm{~s}^{-1}$ \\
\hline
\end{tabular}

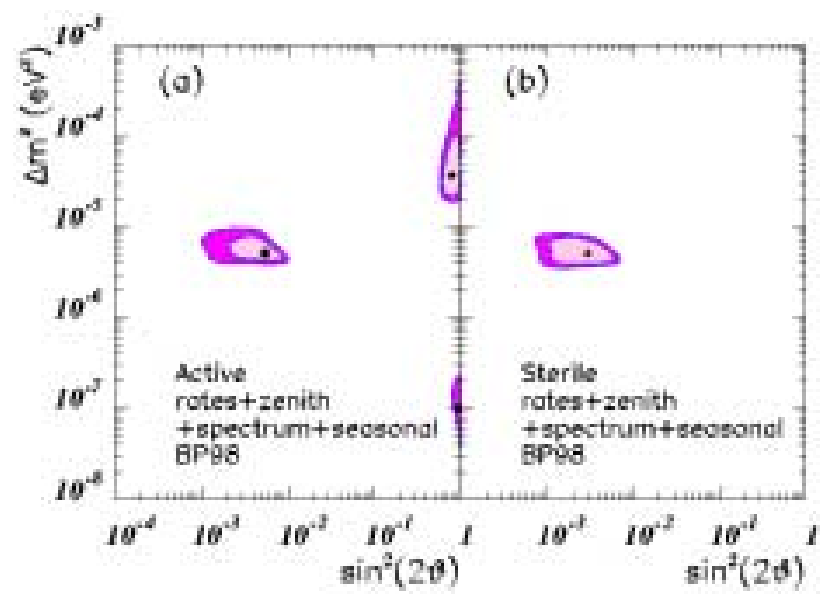

Figure 8. Allowed region for $\sin ^{2} 2 \theta$ and $\Delta m^{2}$ which can explain the observed solar neutrino data. Taken from Ref. [31].

These discrepancies between the observations and theory is called the solar neutrino problem [23, 28] and it is very unlikely that one can modify the SSM such that all the experiments are explained without invoking any non-standard neutrino properties [29]. In Fig. 6 we plot the contours of $\chi^{2}$ corresponding to $1 \sigma, 2 \sigma, \ldots 5$ $\sigma$, assuming that the flux of $p p,{ }^{7} \mathrm{Be}$ and ${ }^{8} \mathrm{~B}$ are treated as a free parameters but only required to be consistent with the observed luminosity of the sun. We see that the data indicate vanishing ${ }^{7} \mathrm{Be}$ neutrino flux with half of SSM ${ }^{8} \mathrm{~B}$ neutrino one even if we neglect any one of the three solar neutrino experiments. This is very difficult to explain by the reasonable modifications of the SSM. 


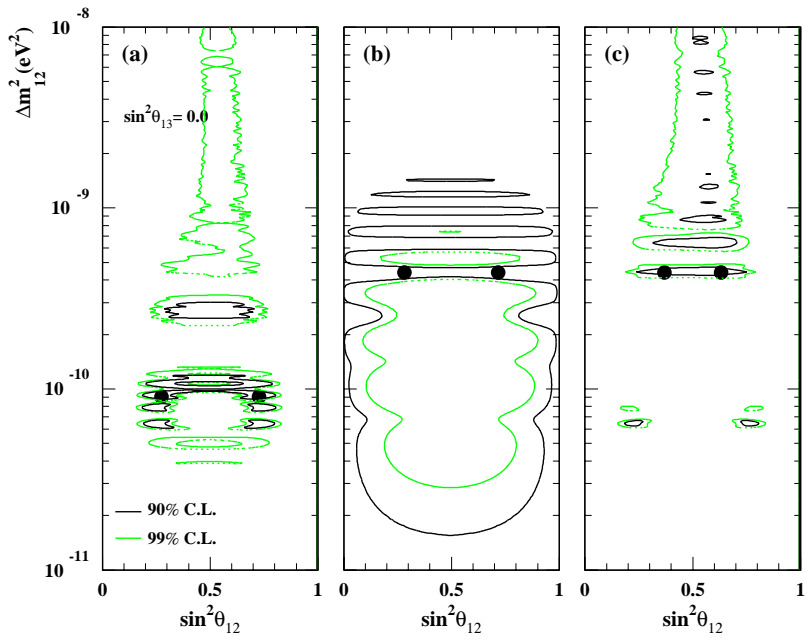

Figure 9. Allowed region for $\sin ^{2} 2 \theta$ and $\Delta m^{2}$ which can explain the observed solar neutrino data by the vacuum oscillation. Taken from Ref. [32].

The most plausible solutions to this problem is provided either by the matter enhanced resonant neutrino conversion, the MSW effect [22] or by the vacuum oscillation [30].

In Fig. 7 we show the MSW allowed region recently obtained in Ref. [31] using total rates and spectrum informations. In Fig. 8 we show similar plots obtained in Ref. [31] but using total rates, spectrum, zenith angle as well as seasonal informations. As we can see in this figure, for the case of active conversion $\nu_{e} \rightarrow \nu_{\mu, \tau}$, there are three separate allowed regions. The one in the smaller mixing angle region is called small mixing angle (SMA) MSW solution, The one in the large mixing angle region with larger $\Delta m^{2}$ is called large mixing angle (LMA) solution and the one in the large mixing angle region with smaller $\Delta m^{2}$ is called large mixing angle is often called low- $\Delta m^{2}$ (LOW) solution. We note in case of sterile conversion, $\nu_{e} \rightarrow \nu_{s}$ there is only SMA MSW solution.

The solar neutrino data can also be explained by the vacuum oscillation between the sun and the earth. In Fig. 9 we show the allowed parameter region determined from (a) total rates (b) SK spectrum and (c) rates + SK spectrum, assuming vacuum oscillation as a solution to the solar neutrino problem. In this scenario, the best fit occurs when the oscillation length for neutrino with energy $\sim 10 \mathrm{MeV}$ is comparable to the sun-earth distance.

\section{Neutrino mass and oscilla- tion search at laboratory}

In this section we review the status of laboratory search for neutrino mass and oscillation.

\section{V.1 Direct mass measurements}

So far, there is no direct evidence of non-zero neutrino mass from the laboratory experiments and we only know that they have much smaller (if non-zero) masses compared to their charged partners. Upper limits from direct kinematic neutrino mass measurements are given as follows [2],

$$
\begin{gathered}
m_{\nu_{e}}<15 \mathrm{eV} \\
m_{\nu_{\mu}}<0.17 \mathrm{MeV} \\
m_{\nu_{\tau}}<18.2 \mathrm{MeV}
\end{gathered}
$$

V.2 Double beta decay Results coming from neutrinoless double beta decay experiment is giving more stringent upper limit on Majorana neutrino mass. The current best limit is coming from the HeidelbergMoscow experiment on $2 \beta$ decay of ${ }^{76} \mathrm{Ge}$ [33],

$$
\left\langle m_{\nu_{e}}\right\rangle \equiv \sum U_{e i}^{2} m_{i}<0.2-0.6 \mathrm{eV}
$$

where $U_{e i}$ is the MNS mixing matrix and $m_{i}$ is mass of $i$-th mass eigenstate.

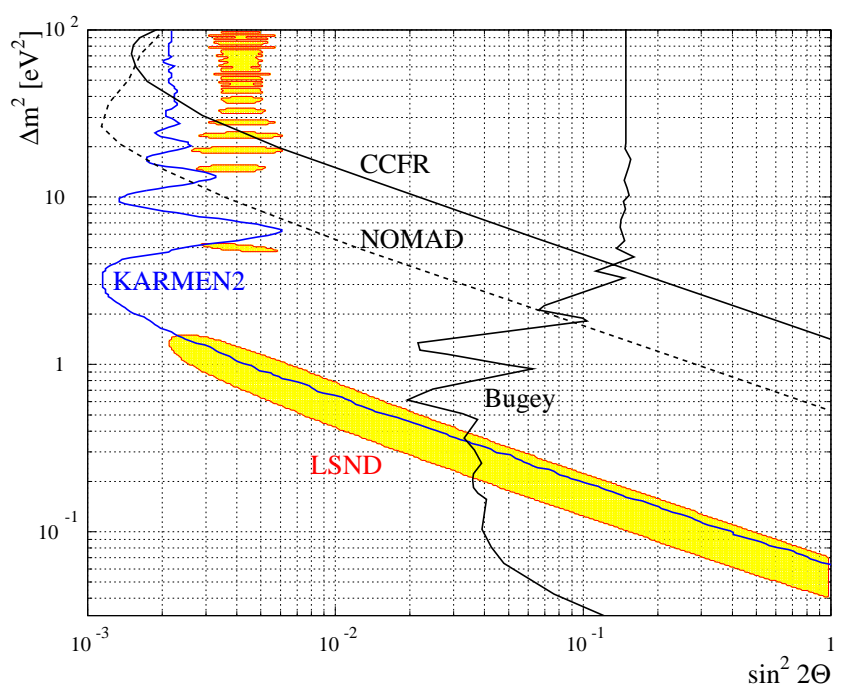

Figure 10. LSND $90 \%$ C. L. region in comparison with other $90 \%$ C. L. exclusion curves in the corresponding $\left(\sin ^{2} 2 \theta, \Delta m^{2}\right)$ plane. Taken from Ref. [34].

\section{V.3 LSND signals}

LSND collaboration has been observing some positive signal of neutrino oscillation of $\bar{\nu}_{\mu} \rightarrow \bar{\nu}_{e}$ with the probability $P\left(\bar{\nu}_{\mu} \rightarrow \bar{\nu}_{e}\right) \sim$ few $\times 10^{-3}$ [15]. This signal can be accounted for by the neutrino oscillation hypothesis if $\Delta m^{2} \gtrsim 0.1 \mathrm{eV}^{2}$ and $\sin ^{2} 2 \theta \gtrsim$ few $\times 10^{-3}$. In Fig. 10 we show the LSND $90 \%$ C.L. together with the exclusion curves from CCFR [35], NOMAD [36], Bugey [37] and KARMEN [38]. 
Although the same type of experiment KARMEN [38] is not observing such positive signal both experiments are still consistent for some parameters of mass and mixing.

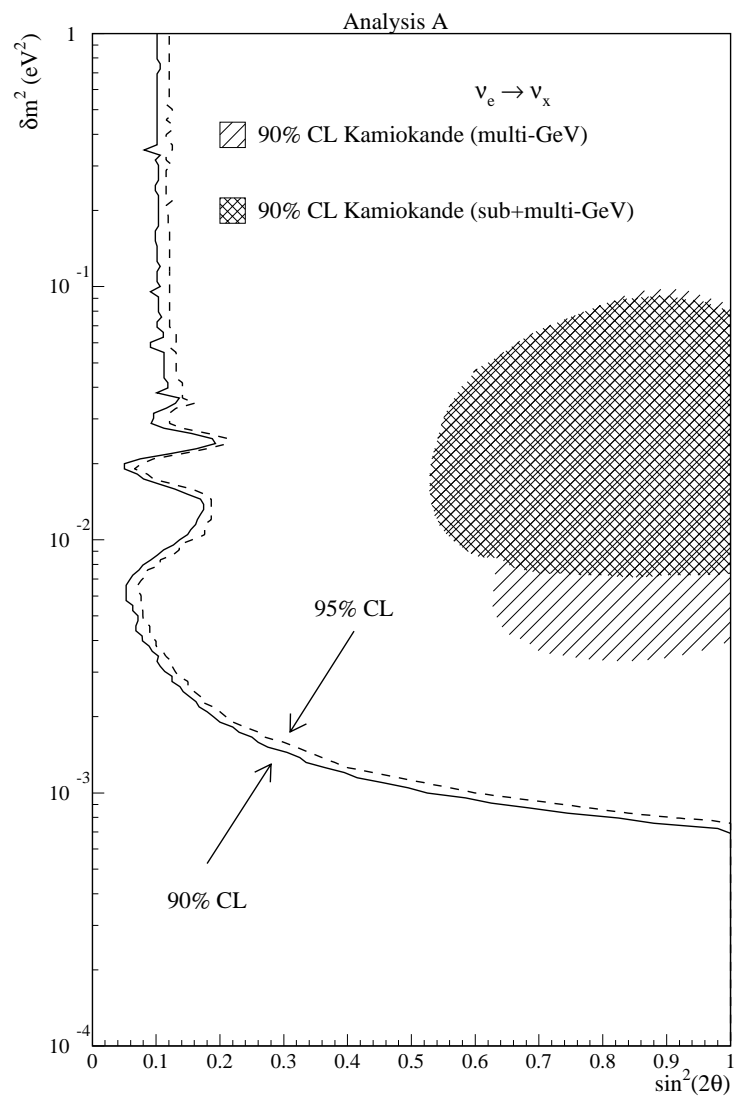

Figure 11. Exclusion plot for the oscillation parameters based on the absolute comparison of measured vs. expected positron yields in the Chooz experiment. Taken from Ref. [41].

\section{V.4 Oscillation search at reactors}

So far, several neutrino oscillation search have been performed by using nuclear reactors [39, 40, 37, 41, 42]. Among them, the most stringent limits on the oscillation parameters have been obtained by the Chooz experiment [41] which excluded the possibility of having large oscillation between $\nu_{e}$ and $\nu_{\mu}$ for atmospheric neutrinos. In Fig. 11 we show the exclusion plot of the oscillation parameters obtained by the Chooz experiment. Similar but some what weaker constraints are obtained from Palo Verde experiment [42].

\section{Multi Flavor Interpretations}

In this section we discuss how the pictures we reviewed in terms of two flavor oscillation in the previous sections could be incorporated into the three or four flavor neutrino scheme.
Let us first summarize below the required values of mass squared differences and mixing angle which are consistent with atmospheric, solar neutrino problem and LSND signal, when analyzed assuming only two flavor mixing,

$$
\begin{gathered}
\text { Atm : } \quad \Delta m_{a t m}^{2} \simeq(2-7) \times 10^{-3} \mathrm{eV}^{2} \\
\sin ^{2} 2 \theta_{a t m} \simeq 0.82-1.0
\end{gathered}
$$

$$
\begin{array}{cc}
\text { MSW SMA : } & \Delta m_{\odot}^{2} \simeq(4-10) \times 10^{-6} \mathrm{eV}^{2} \\
& \sin ^{2} 2 \theta_{\odot} \simeq(0.1-1.0) \times 10^{-2} \\
\text { MSW LMA }: & \Delta m_{\odot}^{2} \simeq(2-20) \times 10^{-5} \mathrm{eV}^{2} \\
& \sin ^{2} 2 \theta_{\odot} \simeq 0.6-0.99
\end{array}
$$

$$
\begin{aligned}
& \text { MSW LOW : } \quad \Delta m_{\odot}^{2} \simeq(6-20) \times 10^{-8} \mathrm{eV}^{2} \\
& \sin ^{2} 2 \theta_{\odot} \simeq 0.9-0.99
\end{aligned}
$$

$$
\begin{gathered}
\mathrm{VO}: \Delta m_{\odot}^{2} \simeq(0.5-10) \times 10^{-10} \mathrm{eV}^{2} \\
\sin ^{2} 2 \theta_{\odot} \simeq 0.6-1.0
\end{gathered}
$$

LSND : $\quad \Delta m_{L S N D}^{2} \simeq(0.2-2) \times \mathrm{eV}^{2}$

$$
\sin ^{2} 2 \theta_{L S N D} \simeq 10^{-3}-0.04
$$

\section{VI.1 Three Flavor Schemes}

It has been noted that only with three flavors, it is impossible to explain atmospheric neutrino anomaly, solar neutrino deficit and LSND signal simultaneously. The reason is simply because we need three different values of mass squared differences or $\Delta m^{2}$ indicated in eqs. (24)- (29) which can not be realized only with three flavors. Therefore, if we assume that there are only three neutrinos we must give up to explain one of the neutrino anomalies.

Neglecting LSND results, if the mixing between the first generation and the third generation is zero or small, $\sin ^{2} \theta_{13} \ll 1$ then we can assign the mixing parameters required to explain atmospheric as well as solar neutrino data in terms of three flavor framework as follows,

$$
\begin{gathered}
\Delta m_{a t m}^{2}=\Delta m_{32}^{2} \simeq \Delta m_{31}^{2} \\
\sin ^{2} 2 \theta_{a t m}=\sin ^{2} 2 \theta_{23}, \\
\Delta m_{\odot}^{2}=\Delta m_{21}^{2}, \\
\sin ^{2} 2 \theta_{\odot}=\sin ^{2} 2 \theta_{12}
\end{gathered}
$$

where $\Delta m_{i j}^{2} \equiv m_{i}^{2}-m_{j}^{2}$.

It can be shown that for the observations of solar and atmospheric neutrinos with the mixing parameters indicated in eqs. (24)-(28), the CP violating phase $\delta$ does not play any important role and we can simply neglect it even if $\theta_{13}$ is large. If $\theta_{13}$ is zero or sufficiently small, one can fit separately assuming only 
two flavors the solar neutrino and atmospheric neutrino data and the results shown in the previous sections in terms of two neutrino flavor are valid. Observationally, oscillations of atmospheric and solar neutrinos do not interfere each other because of the mass hierarchy $\Delta m_{\text {atm }}^{2} \gg \Delta m_{\odot}^{2}$ and the fact that $\nu_{\mu}$ and $\nu_{\tau}$ equally contribute to solar neutrino detection at Kamiokande and Super-Kamiokande. Oscillation between $\nu_{\mu}$ and $\nu_{\tau}$ is irrelevant for solar neutrino observations.

Below let us try to discuss the possible mixing patterns.

\section{(i) Only one large mixing}

In order to explain the atmospheric neutrino data, under the assignments of the parameters in eq. (31), we assume that $\theta_{23}$ and $\Delta m_{32}^{2}$ are in the range indicated in eq. (24). Then atmospheric neutrino data can be explained by the large vacuum oscillation between $\nu_{\mu}$ and $\nu_{\tau}$ due to large $\theta_{23}$. If we further assume that, under the assignments of the parameters in eq. (31), $\theta_{21}$ and $\Delta m_{21}^{2}$ are in the range indicated in eq. (25), then solar neutrino problem can be explained by the small mixing angle MSW solution, without affecting the oscillation interpretation of the atmospheric neutrino between $\nu_{\mu}$ and $\nu_{\tau}$. In this case $\nu_{1}$ is mostly $\nu_{e}$ and has small component of $\nu_{\mu}$ and $\nu_{\tau}$ and the solar $\nu_{e}$ 's are resonantly converting into $\nu_{2}$, which is the mixed state of $\nu_{\mu}$ and $\nu_{\tau}$, by making use of the mass difference of $\nu_{1}$ and $\nu_{2}$ states. This picture is graphically presented in Fig. 12 .

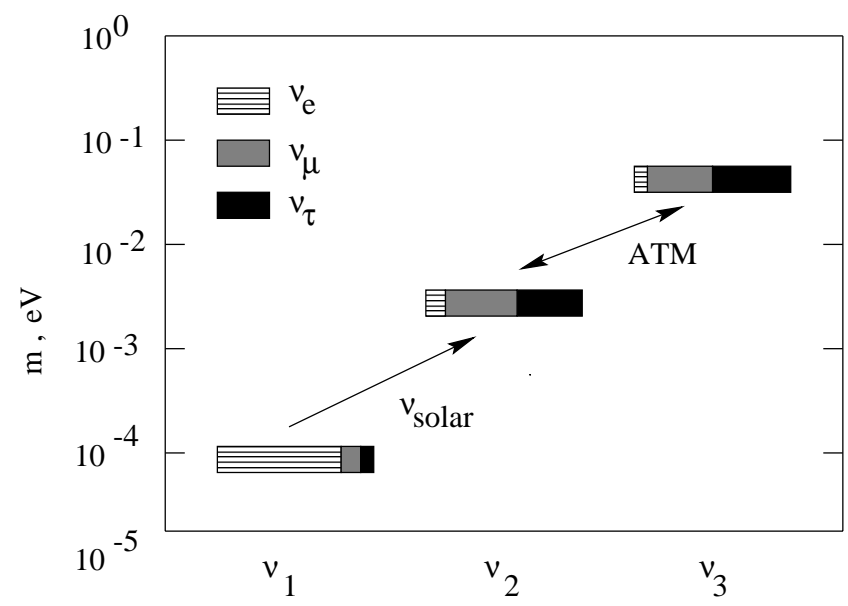

Figure 12. 3-flavor schemes of neutrino masses and mixing. Solutions of the solar neutrino problem is SMA MSW. Taken from Ref [43].

\section{(ii) Two large mixing}

As in the first case in (i), we assume that $\theta_{23}$ and $\Delta m_{32}^{2}$ are in the range indicated in eq. (24) so that atmospheric neutrino can be explained by the large vacuum oscillation between $\nu_{\mu}$ and $\nu_{\tau}$. If we further assume $\theta_{21}$ and $\Delta m_{21}^{2}$ are in the range indicated in eq. (26) or (27), then solar neutrino problem can be explained by the large mixing angle MSW solution, again without affecting the oscillation interpretation of the atmospheric neutrino oscillation. If $\theta_{21}$ and $\Delta m_{21}^{2}$ are in the range in eq. (28), then the solar neutrino problem can be explained by the vacuum oscillations. In these scenarios, $\nu_{e}$ is strongly mixed with $\nu_{\mu}$ and $\nu_{\tau}$ but the oscillation between $\nu_{e}$ and other flavor is negligible for atmospheric neutrinos because of the small values of $\Delta m_{21}^{2}$. These two pictures are graphically shown in Figs. 13 and 14.

\section{(ii) Large $\theta_{13}$ ?}

If $\theta_{13}$ is not so small then the simple pictures described above must be modified. The relevant values of the mass and mixing parameters in order to fit the atmospheric and solar neutrino data could be significantly different from the ones indicated in eqs. (24)- (28) as were demonstrated in Ref. [44] for atmospheric neutrino and Ref. [45, 32] for solar neutrino observations. However, we note that $\theta_{13}$ can not be so large due to the constraint from Chooz [41] and Super-Kamiokande atmospheric neutrino data itself [7], and the pictures described in (i) and (ii) basically hold.

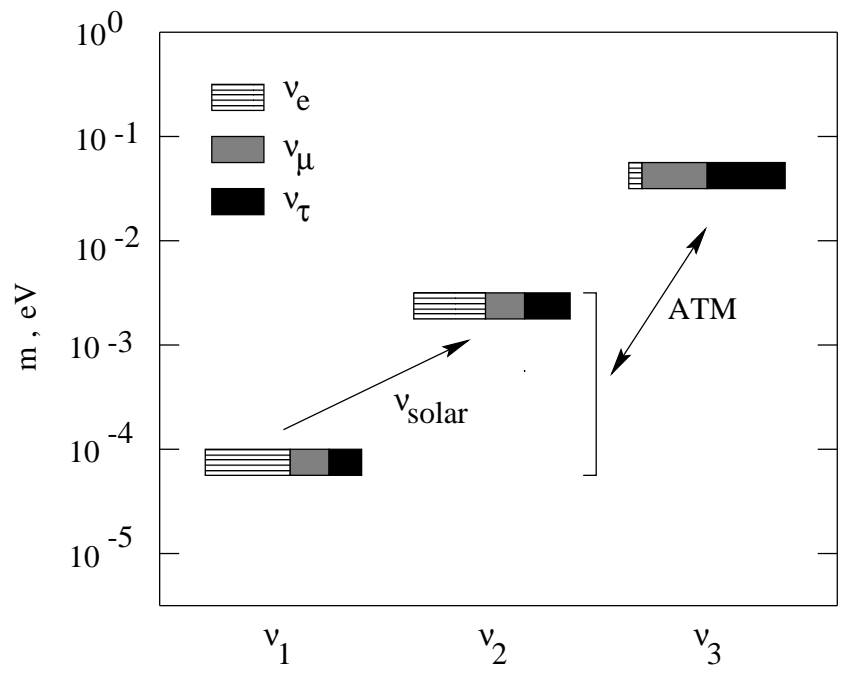

Figure 13. 3-flavor schemes of neutrino masses and mixing. Solutions of the solar neutrino problem is LMA MSW. Taken from Ref [43].

\section{VI.2 Four Flavor Scheme}

If we want to explain also the LSND signal, in addition to the atmospheric and solar neutrino anomaly, it is necessary to introduce fourth neutrino, which must be sterile (electroweak singlet) which can only have much weaker interactions than usual neutrinos [46]. The currently most favored picture is as follows. $\nu_{\mu}$ and $\nu_{\tau}$ are strongly mixed so that atmospheric neutrino data can be explained by the large vacuum oscillations between them. $\nu_{e}$ is weakly mixed with $\nu_{s}$ (sterile) neutrino in such a way that the solar neutrino problem can 
be explained by the small angle MSW solution with $\nu_{e} \rightarrow \nu_{s}$ channel. Further $\nu_{e}$ is weakly mixed with $\nu_{\mu}$ with adequate mass squared difference so that LSND data can be explained. This scheme can also provide some amount of hot dark matter which are mainly $\nu_{\mu}$ and $\nu_{\tau}$. There is also some astrophysical consideration that this scheme is also consistent with the hypothesis of heavy elements nucleosynthesis in the supernova explosion [47]. This picture is graphically presented in Fig. 15.

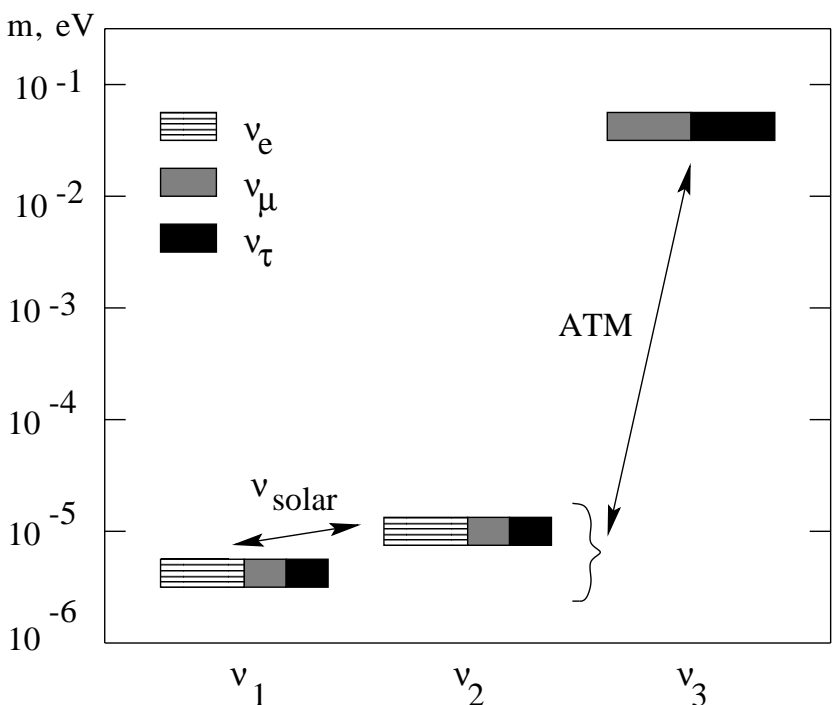

Figure 14. 3-flavor schemes of neutrino masses and mixing. Solution of the solar neutrino problem is VO. Taken from Ref [43].

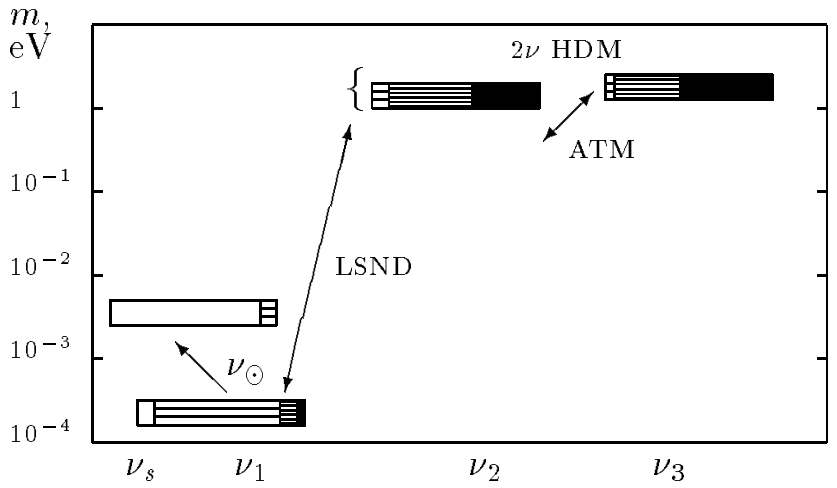

Figure 15. 4-flavor schemes of neutrino masses and mixing. Solutions of the solar neutrino problem is SMA MSW $\nu_{e} \rightarrow \nu_{s}$ conversion. Taken from Ref [48].

\section{Conclusions and Outlook}

As we have seen that there are several strong indications of neutrino mass and flavor mixing. Although they are sufficiently strong, neutrino mass and flavor mixing have not yet been really established and therefore, must be confirmed by the up coming experiments.
This is important also to exclude the other possibilities which are not discussed in this review. We note that there are still another possible explanations of the neutrino anomalies which do not use the usual flavor mixing. It has been proposed that atmospheric neutrino observations could be explained by neutrino decay $[49,50]$ or flavor changing interactions in matter [51] though scenarios in Refs. [49, 51] have been criticized and disfavored by the discussion in Ref. [52]. See also Refs. [53, 54, 55, 56] for the scenario with flavor changing interactions. Solar neutrino problem could be explained by resonant spin-flavor precession [57, 58], flavor changing interactions $[59,60]$ or even by a tiny violation of equivalence principle [61]. The above possibilities are not yet excluded and have to be refuted by the experiments, before the establishment of the neutrino mass and flavor mixing.

For the purpose of the confirmation (or refutation) of the oscillation interpretations discussed in this review, several new experiments are planned. To confirm the atmospheric neutrino observations, the first long-baseline (LBL) neutrino oscillation experiment K2K [62] with baseline $L=250 \mathrm{~km}$, sending neutrino beam from KEK to the Super-Kamiokande detector, is already started to take data and some results will be reported very soon. There is also another LBL experiment called MINOS [63] with baseline $L=732 \mathrm{~km}$, from Fermilab to Soudan mine.

For solar neutrinos, new generation experiment, Sudbury Neutrino Observatory (SNO) solar neutrino experiment [64] which uses heavy water (deuteron) is already taking data and will have some results soon. One of the feature of this experiment is that they can measure separately the event from the charged current reaction, $d+\nu_{e} \rightarrow p+p+e^{-}$and the one from the neutral current reaction $d+\nu_{x} \rightarrow n+p+\nu_{x}(x=e, \mu, \tau)$ so that they can confirm if the active to active neutrino conversion $\nu_{e} \rightarrow \nu_{\mu, \tau}$ is occurring. See, for e.g., Ref. [65] for the recent discussion on the potential power of the SNO experiment.

It is also very important to observe ${ }^{7} \mathrm{Be}$ neutrinos to establish neutrino oscillation hypothesis. For this purpose, several experiments such as BOREXINO [66], KamLAND [67] are planned. We also note that KamLAND can confirm/refute the LMA MSW solution by observing the neutrinos from nearby reactors. There are also some experiments which can identify the lower energy neutrinos coming from $p p$ reactions, HELLAZ [68] and HERON [69].

In order to confirm/refute LSND signal, the MiniBOONE experiment [70], which can cover completely the LSND allowed region shown in Fig. 10, will begin data taking in the year 2002 .

We are now definitely entering into new era for neutrino physics and in the next decade, various very im- 
portant informations will be obtained.

\section{Acknowledgments}

The author was supported by Fundação de Amparo à Pesquisa do Estado de São Paulo (FAPESP). The author would like to thank M. M. Guzzo, P. C. de Holanda, R. Z. Funchal and other members of GEFAN for useful discussions.

\section{References}

[1] T. Kafka for E872 collaboration, Nucl. Phys. (Proc. Suppl.) B 70, 204 (1999).

[2] C. Caso et al., Eur. Phys. J. C 3, 1 (1998).

[3] IMB Collaboration, D. Casper et al., Phys. Rev. Lett. 66, 2561 (1991); R. Becker-Szendy et al., Phys. Rev. D 46, 3720 (1992).

[4] Kamiokande Collaboration, H. S. Hirata et al., Phys. Lett. B 205, 416 (1988) and Phys. Lett. B 280, 146 (1992); Kamiokande Collaboration, Y. Fukuda et al., Phys. Lett. B 335, 237 (1994)

[5] Soudan Collaboration, W. W. M Allison et al., Phys. Lett. B 391, 491 (1997); T. Kafka for Soudan Collaboration, hep-ex/9912060.

[6] Macro Collaboration, M. Ambrosio et al., Phys. Lett. B 434, 451 (1998); hep-ex/0001044

[7] SuperKamiokande Collaboration, Y. Fukuda et al., Phys. Lett. B 433, 9 (1998); Phys. Rev. Lett. 81, 1562 (1998); Phys. Lett. B 436, 33 (1998); Phys. Rev. Lett. 82, 2644 (1999); Phys. Lett. B 467, 185 (1999).

[8] K. Scholberg, hep-ex/9905016.

[9] W. A. Mann, hep-ex/9912007.

[10] Homestake Collaboration, K. Lande et al., Astrophys .J. 496, 505 (1998);

[11] Sage Collaboration, J. N. Abdurashitov et al., Phys. Rev. C 60, 055801 (1999);

[12] GALLEX Collaboration, W. Hampel et al., Phys. Lett. B 447, 127 (1999);

[13] Y. Fukuda et al. (Kamiokande Collaboration), Phys. Rev. Lett. 77, 1683 (1996).

[14] SuperKamiokande Collaboration, Y. Fukuda et al., Phys. Rev. Lett. 81, 1158 (1998); ibid. 81, 4279 (1998); ibid. 82, 2430 (1999); ibid. 82, 1810 (1999).

[15] C. Athanassopoulos et al. , for LSND collaboration, Phys. Rev. Lett. 77, 3082 (1996); Phys. Rev. C54, 2685 (1996).

[16] J. A. Holtzman, Astrophys. J. Suppl. 71, 1 (1989); J. A. Holtzman and J. R. Primack, Astrophys. J. 405, 428 (1993); J. R. Primack, J. Holtzman, A. Klypin, and D. O. Caldwell, Phys. Rev. Lett. 74, 2160 (1995); K. S. Babu, R. K. Schaefer, and Q. Shafi, Phys. Rev. D53, 606 (1996); D. Pogosyan and A. Starobinsky, astro-ph/9502019.

[17] B. Pontecorvo, Sov. Phys. JETP 26, 984 (1968).
[18] Z. Maki, M. Nakagawa and S. Sakata, Prog. Theor, Phys. 28, 870 (1962).

[19] N. Cabbibo, Phys. Rev. Lett. 10, 531 (1963); M. Kobayashi and T. Maskawa, Prog. Theor. Phys. 49, 652 (1973).

[20] M. Fukugita and T. Yanagida, in Physics and Astrophysics of Neutrinos edited by M. Fukugita and A. Suzuki, Springer Verlag, 1994.

[21] See, e.g., M. Tanimoto, Phys. Rev. D55, 322 (1997); Prog. Theo. Phys. 97, 901 (1997); J. Arafune and J. Sato, Phys. Rev. D55, 1635 (1997); J. Arafune, M. Koike and J. Sato, Phys. Rev. D56, 3093 (1997); H. Minakata and H. Nunokawa, Phys. Rev. D57, 4403 (1998); Phys. Lett. B413, 369 (1998); M. Bilenky, C. Giunti, W. Grimus, Phys. Rev. D58, 033001 (1998); A. Gago, V. Pleitez and R. Zukanovich Funchal, Phys. Rev. D61, 016004 (2000); A. Donini, M.B.Gavela, P. Hernandez and S. Rigolin, hep-ph/9910516; M. Koike and J. Sato, hep-ph/9911258.

[22] M. Mikheyev, A. Smirnov, Sov. J. Nucl. Phys. 42, 913 (1986); L. Wolfenstein, Phys. Rev. D17, 2369 (1978); Phys. Rev. D 20, 2634 (1979).

[23] J. N. Bahcall, Neutrino Astrophysics, Cambridge University Press, Cambridge, England, 1989; see also J.N. Bahcall's web site, http://www.sns.ias.edu/ jnb.

[24] C-S. Lim, Preprint, BNL-39675.

[25] Fréjus Collaboration, Ch. Berger et al., Phys. Lett. B 227, 489 (1989).

[26] NUSEX Collaboration, M. Aglietta et al., Europhys. Lett. 8, 611 (1989).

[27] J.N. Bahcall, S. Basu and M.H. Pinsonneault, Phys. Lett. B 433, 1 (1998).

[28] J. N. Bahcall, hep-ex/0002018.

[29] For a recent discussion, see for e.g., H. Minakata and H. Nunokawa, Phys. Rev. D 59, 073004 (1999) and references therein.

[30] V. N. Gribov and B. M. Pontecorvo, Phys. Lett. B 28, 493 (1969)

[31] M.C. Gonzalez-Garcia, P.C. de Holanda, C. PeñaGaray and J.W.F. Valle, hep-ph/9906469, Nucl. Phys. $\mathrm{B}$, in press.

[32] A. Gago, H. Nunokawa and R. Zukanovich Funchal, in preparation.

[33] L. Baudis et al., Phys. Rev. Lett. 83, 41 (1999).

[34] K. Eitel, New Jour. Phys. 2, 1.1-1.25 (2000) [hepex/9909036].

[35] A. Romosan et al., Phys. Rev. Lett. 78, 2912 (1997).

[36] M. Mezzetto et al, Nucl. Phys. (Proc. Suppl.) B 70, 214 (1999).

[37] B. Achkar et al., Nucl. Phys. B 434, 503 (1995).

[38] K. Eitel and B. Zeitnitz, for KARMEN collaboration, Nucl. Phys. B (Proc. Suppl.) 77, 212 (1999).

[39] G. Zacek et al., Phys. Rev. D 34, 2621 (1986).

[40] G.S. Vidyakin et al., Pis'ma Zh. Eksp. Teor, Fiz. 59, 364 (1994) [JETP Lett. 59, 390 (1994)]. 
[41] CHOOZ Collaboration, M. Apollonio et al., Phys. Lett. B 420, 397 (1998); ibid., B 466, 415 (1999).

[42] Palo Verde Collaboration, F. Boehm et al., hepex/9912050.

[43] A. Yu Smirnov, hep-ph/9907296.

[44] G.L.Fogli, E.Lisi, D. Montanino, G.Scioscia, Phys. Rev. D 55, 4385 (1997); G.L.Fogli, E.Lisi, A. Marrone, G.Scioscia, Phys. Rev. D 59, 033001 (1999); O. Yasuda, Phys. Rev. D 58, 091301 (1998).

[45] G. L. Fogli, E. Lisi and D. Montanino, Physical Review D 50, 2048 (1996); G. L. Fogli, E. Lisi, D. Montanino and A. Palazzo, hep-ph/9912231.

[46] J. T. Peltoniemi, D. Tommasini, and J. W. F. Valle, Phys. Lett. B 298, 383 (1993); J. T. Peltoniemi and J. W. F. Valle, Nucl. Phys. B 406, 409 (1993); D. Caldwell and R. N. Mohapatra, Phys. Rev. D 50, 3477 (1994); G. M. Fuller, J. R. Primack and Y.-Z. Qian, Phys. Rev. D 52, 1288 (1995); J. J. Gomez-Cadenas and M. C. Gonzalez-Garcia, Zeit. fur Physik C 71, 443 (1996); E. Ma and P. Roy, Phys. Rev. D 52, R4780 (1995); E. Ma and J. Pantaleone, Phys. Rev. D 52, R3763 (1995); R. Foot and R. R. Volkas, Phys. Rev. D 52, 6595 (1995); Z. G. Berezhiani and R. N. Mohapatra, Phys. Rev. D 52, 6607 (1995); E. J. Chun, A. S. Joshipura and A. Y. Smirnov, Phys. Lett. B 357, 608 (1995); N. Okada and O. Yasuda, Int. J. Mod. Phys. A 12, 3669 (1997); Q. Y. Liu and A. Yu. Smirnov, Nucl. Phys. B 524, 505 (1998); A. Joshipura and A. Yu. Smirnov, Phys. Lett. B 439, 103 (1998); S. C. Gibbons, R. N. Mohapatra, S. Nandi and A. Raychaudhuri, Phys. Lett. B 466, 115 (1999).

[47] D. O. Caldwell, G.M. Fuller and Y-Z. Qian, astro$\mathrm{ph} / 9910175$.

[48] A. Yu Smirnov, hep-ph/9611465.

[49] V. Barger, J.G. Learned, S. Pakvasa and T.J. Weiler, Phys. Rev. Lett. 82, 2640 (1999).

[50] V. Barger et al., Phys. Lett. B 462, 109 (1999).

[51] M. C. Gonzalez-Garcia et al., Phys. Rev. Lett. 82, 3202 (1999).

[52] P. Lipari and M. Lusignoli, Phys. Rev. D 60, 013003 (1999).

[53] N. Fornengo, M.C. Gonzalez-Garcia and J. F. W. Valle, hep-ph/9906539.

[54] M. M. Guzzo et al., to be published in the proceedings of TAUP99.

[55] S. Bergmann, Y. Grossman and D. M. Pierce, Phys. Rev. D 61, 053005 (2000); M. M. Guzzo,
H. Nunokawa, O. L. G. Peres, V. Pleitez and R. Zukanovich Funchal, hep-ph/9908308.

[56] A. M. Gago, L. P. Freitas, O. L. G. Peres, and R. Zukanovich Funchal, hep-ph/9911470.

[57] C.S. Lim and W.J. Marciano,Phys. Rev. D 37, 1368 (1988);

E. Akhmedov and M.Y. Khlopov, Mod. Phys. Lett. A 3, 451 (1988);

E. Akhmedov, Sov. J. Nucl. Phys. 48, 382 (1988); Phys. Lett. B 213, 64 (1988).

[58] For a recent analysis, see for e.g., M.M. Guzzo and H. Nunokawa, Astropart. Phys. 12, 87 (1999).

[59] M.M. Guzzo, A. Masiero and S.T. Petcov, Phys. Lett. B 260, 154 (1991).

[60] S. Bergmann, M.M. Guzzo, P.C. de Holanda, H. Nunokawa and P.I. Krastev, in preparation.

[61] J. Pantaleone, A. Halprin, and C. N. Leung, Phys. Rev. D 47, R4199 (1993); J. N. Bahcall, P. I. Krastev, and C. N. Leung, Phys. Rev. D 52, 1770 (1995); S. W. Mansour and T. K. Kuo, Phys. Rev. D 60, 097301 (1999); A. M. Gago, H. Nunokawa and R. Zukanovich Funchal, hep-ph/9909250, to be published in Phys. Rev. Lett.

[62] K. Nishikawa, Nucl. Phys. (Proc. Suppl.) B 77, 198 (1999); Y. Oyama, hep-ex/9803014.

[63] B.C. Barish, Nucl. Phys. (Proc. Suppl.) B 70, 227 (1999); S.G. Wojcicki, Nucl. Phys. (Proc. Suppl.) B 77, 182 (1999).

[64] A.B. McDonald, for the SNO collaboration, Nucl. Phys. (Proc. Suppl.) B 77, 43 (1999).

[65] J.N. Bahcall, P.I. Krastev and A.Yu. Smirnov, Phys. Lett. B 477, 401 (2000); hep-ph/0002293.

[66] L. Oberauer, Nucl. Phys. (Proc. Suppl.) B 77, 48 (1999).

[67] A. Suzuki, Nucl. Phys. (Proc. Suppl.) B 77, 171 (1999).

[68] A. De Bellefon for HELLAZ collaboration, Nucl. Phys. (Proc. Suppl.) B 70, 386 (1999).

[69] R. E. Lanou, in Proceedings of the 8th International Workshop on Neutrino Telescopes (Venice, Italy, 1999), ed. by M. Baldo Ceolin, Vol. I, page 139.

[70] E. Church et al., A proposal for an experiment to measure $\nu_{\mu} \rightarrow \nu_{e}$ oscillations and $\nu_{\mu}$ disappearance at the Fermilab Booster: BOONE, 7 December 1997 (unpublished). 